\title{
A discrete dynamical system for the Marx model
}

\author{
Henrique Oliveira \& Rafael Luís
}

To cite this article: Henrique Oliveira \& Rafael Luís (2011) A discrete dynamical system for the Marx model, Journal of Difference Equations and Applications, 17:8, 1199-1206, DOI: $10.1080 / 10236190903052888$

To link to this article: https://doi.org/10.1080/10236190903052888

\section{曲 Published online: 30 Mar 2011.}

Submit your article to this journal $ऍ$

Шll Article views: 174

Q View related articles $₫$

47 Citing articles: 1 View citing articles 지 


\title{
A discrete dynamical system for the Marx model
}

\author{
Henrique Oliveira ${ }^{\mathrm{a} *}$ and Rafael Luís ${ }^{\mathrm{b} 1}$ \\ ${ }^{a}$ Department of Mathematics, Instituto Superior Técnico, Technical University of Lisbon, Lisbon, \\ Portugal; ${ }^{b}$ Center for Mathematical Analysis, Geometry, and Dynamical Systems, Instituto Superior \\ Técnico, Technical University of Lisbon, Lisbon, Portugal
}

(Received 3 April 2009; final version received 16 May 2009)

\begin{abstract}
The Marx model for the profit rate $r$ depending on the exploitation rate $e$ and on the organic composition of the capital $k$ is studied using a dynamical approach. Introducing a discrete dynamical system in the model and supposing that both $k$ and $e$ depend on the profit rate of the previous cycle we get a discrete dynamical system for $r, r_{n+1}=f_{a}\left(r_{n}\right)$, which is a family of unimodal maps depending on the parameter $a$, where $a$ is the exploitation rate at profit zero. It is interesting to note that the model can model the phenomenon known as Kondratiev waves in economic systems.
\end{abstract}

Keywords: Marx model; profit rate; exploitation rate; organic composition of the capital; Kondratiev waves

\section{Introduction}

We find in [4] a well-known equation from economic theory. In this work we will deduct the model and study it in terms of dynamical systems theory.

The equation quoted above describes the behaviour of the profit rate $r$, with the exploitation rate $e$ and with the organic composition of the capital $k$, in the Marx model. The study of this equation in a dynamical system perspective seems to be very interesting, because it relates the magnitudes $r, e$ and $k$ by the equation

$$
r=\frac{e}{1+k}
$$

Our main goal is to use this equation as the starting point of a study in which we may model $e(t), k(t)$ and $r(t)$ as variables depending on $t$, using the formalism of discrete dynamical systems.

In the second section we introduce the economic variables that permit to deduct relation (1). In the third section we introduce in the model an iterative approach: the organic composition of the capital and the exploitation rate are now dependent on the same variable (profit rate) in the previous cycle. We now have discrete variables and the system starts to react to what happened before. In this section we will also introduce a concrete model, that, like many other possible models, may describe the dynamical situation in a realistic way. We prove that, in certain cases, this approach may lead to stability situations with well-defined limits, may lead to periodic orbits (where the profit rate repeats itself from $p$ to $p$ units of time) or may lead to chaos [3] where, in this case, it is impossible to

\footnotetext{
*Corresponding author. Email: holiv@math.ist.utl.pt
} 
predict the evolution of the system. In this last case, the system becomes strongly dependent on the initial conditions but we do not consider this case in this paper.

In the last section using a discrete dynamical system we study the profit rate in the long term. It is interesting to note that using a scale of 10 years, our system exhibits the behaviour known in economy as Kondratiev waves. Moreover, according with this theory, in the current decade we are experiencing the winter season. This situation is in concordance with the crises that the global economic system presents today.

In this work we prove that the Marx model is open to several mathematical approaches and, depending on which approach we use, the conclusion may go from decrease, to stability, to periodicity and even to chaos of the profit rate.

It is wrong to say that this subject is closed by saying that it leads to an indetermination. Quite to the contrary, this model is still open and may be treatable mathematically with profitable economic interpretations.

\section{The Marx model for the economy}

In this section, we introduce a breve description of the Marx model for the profit rate. We present a simple derivation to get the model.

In order to find the relation for the profit rate let us remember that, according to Marx

$$
\text { Profit rate }=\frac{\text { obtained profit }}{\text { invested capital }}
$$

where

$$
\text { Invested capital }=\text { constant capital }+ \text { human capital. }
$$

We also know that

$$
\text { Exploitation rate }=\frac{\text { obtained profit }}{\text { human capital }}
$$

and

$$
\text { Organic composition of the capital }=\frac{\text { constant capital }}{\text { human capital }} \text {. }
$$

Simplifying the relation for the profit rate we obtain

$$
\text { Profit rate }=\frac{\text { exploitation rate }}{1+\text { organic composition of the capital }}
$$

which is equation (1)

For more elaborated details with this subject see [2,3].

\section{Introduction of a discrete dynamical system in the Marx model}

In this section, we will construct a discrete dynamical model for the profit rate, considering that the profit rate for the current cycle is obtained using the exploitation rate and the organic composition of the capital as functions of the profit rate from the time unit immediately before. 
In the economic world of today any decision is conditioned by what happened immediately before. That is, we can suppose that the exploitation rate and the organic composition of the capital depend on the profit rate obtained in the time instant immediately before. The time unit in this case can be, in general, a year. Therefore, $n+1$ represents the year after the current year $n$. The variable in the year $n+1$ will be a function of the variable of the year $n$. It is obvious that we can consider another time unit, for example, a month or a decade, depending of the rate of change in the system.

Therefore the magnitudes in the $(n+1)$ th iteration are functions that depend on the $n$th iteration. We consider these functions continuous and differentiable. We have

$$
e_{n+1}=E\left(r_{n}\right), \quad k_{n+1}=K\left(r_{n}\right) .
$$

In this case the profit rate depends also on the profit rate of the year before. By replacing the relations (2) in (1), we obtain

$$
r_{n+1}=\frac{E\left(r_{n}\right)}{1+K\left(r_{n}\right)}
$$

Now we will introduce a dynamical system considering the economic universe as a whole. We are not interested in considering a specific country or company.

The model that we propose is based on the following assumptions:

(1) We suppose that we have not had any losses, i.e. when we consider the economy as a whole, we are supposing that a negative profit rate does not exist. In fact, we think that in all of the economic activity there exists a positive balance (possibly zero) of the profits. On the other hand, the profit rate cannot be unlimited because that contradicts the fact that the total quantity of money in the planet is finite.

(2) When the profit rate is low, the exploitation tends to be high. In general, when the companies are created or when they face financial difficulties it is very common for the workers to make an extra effort to help the company to obtain or regain satisfactory results. Consequently we will consider that the exploitation rate is high when the profit is low.

On the other hand, when the profit rate is high, the pressure on workers tends to decrease and therefore the exploitation rate tends to decrease. We will consider here that when the profit rate is very large the exploitation rate is zero. So, we consider the following linear model for the exploitation rate

$$
E\left(r_{n}\right)=\left\{\begin{array}{l}
a\left(b-r_{n}\right) \text { if } r_{n} \in[0, b], \\
0 \text { if } r_{n}>b
\end{array}\right.
$$

based on the assumption mentioned above, where $a$, the exploitation at null profit, is a positive real value and $b$ is an adaptable parameter that adjusts to the economic situation.

Therefore, for the study of a concrete model, let us choose $b=1.7$. Having the previous assumptions in mind, we could have chosen another value. It is clear that this value can be adaptable to the economic information available. On the other hand, the real parameter $a$ has strongly influence in the exploitation rate when profits are low, but when profits are high it will have a low influence. In Figure 1, we can see some examples. 


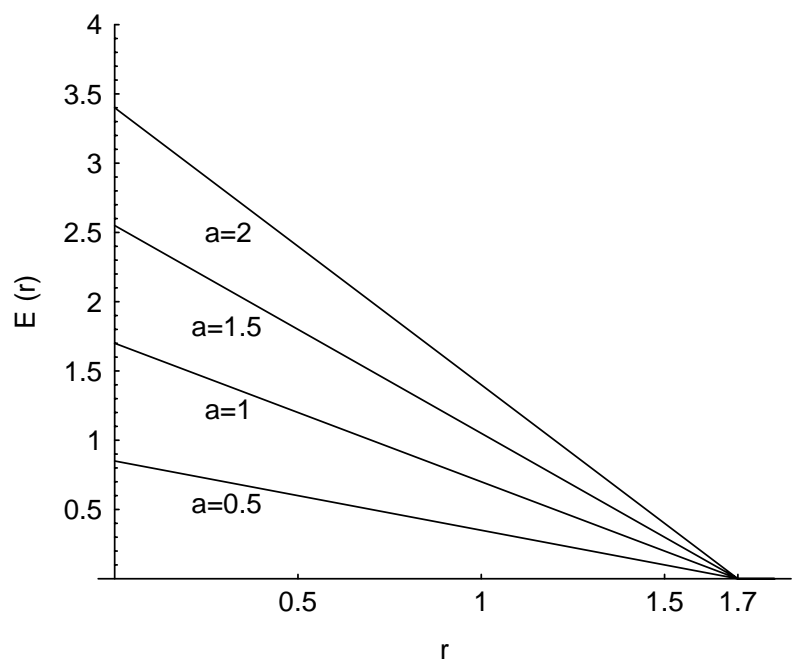

Figure 1. The evolution of the exploitation rate when $a=0.5,1,1.5$ and 2 .

(3) If the economic system has a low profit rate, the trend will be to incorporate more capital (invest) and, on the other hand, to decrease the human capital, through dismissals, that will lead to an increase of the organic composition of the capital. If the profit is very high, the trend will be to reinvest in the capital: human capital (technical formation) and constant capital (technological innovation). A model that describes this reality is given by a capital function $K$ depending on the profit rate of the previous cycle, that is,

$$
K\left(r_{n}\right)=\left\{\begin{array}{l}
\frac{0.05}{\left(r_{n}+0.001\right)\left(b-r_{n}\right)} \quad \text { if } \quad r_{n} \in[0, b] \\
0 \quad \text { if } \quad r_{n}>b
\end{array}\right.
$$

The value of $b$ in our model was considered equal to 1.7. The value 0.001 is used to give us a reasonable value for the capital when the profit is low, and the value 0.05 will originate an equilibrium in the organic composition of the capital, between low profit and high profit rate. We emphasize the fact that when the profit is high, i.e. for values near to 1.7 , the organic composition of the capital as a function of the profit rate, grows unlimitedly, which is acceptable because the profit is always bounded. We can see in Figure 2, a representation of the function $K(r)$.

By replacing the relations (4) and (5) in (3), we obtain for the profit rate

$$
r_{n+1}=\left\{\begin{array}{l}
\frac{a\left(b-r_{n}\right)^{2}\left(r_{n}+0.001\right)}{\left(r_{n}+0.001\right)\left(b-r_{n}\right)+0.05} \quad \text { if } r_{n} \in[0, b] \\
0 \quad \text { if } r_{n}>b
\end{array}\right.
$$

In Figure 3, we see an instance of the plot for the profit rate defined in 6.

For values $0<a<1.0690451$ this iteration has a stable fixed point. For example, for $a=0.75$, the profit rate tends to 0.70004 . When $1.0690451<a<1.2267617$ we do not have a stable fixed point, but oscillations between two points. For example, for $a=1.1$ the profit rate oscillates between 0.376535 and 1.32338 . If the parameter increases more, 


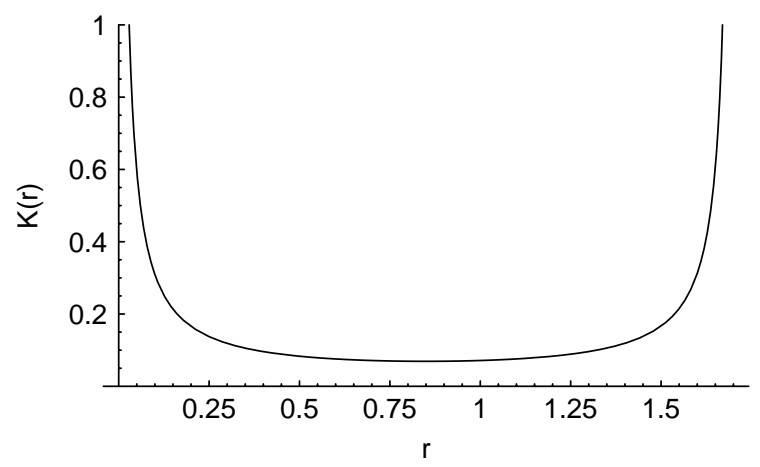

Figure 2. The evolution of the organic composition of the capital when we increase the profit rate.



Figure 3. A prototype of the profit rate when $a=1.5$.

we would have an even more complex situation, with period doubling until the system turns completely unpredictable [3]. For $1.3204<a<1.37291, r_{n}$ has orbits of period five. When the parameter $a$ grows even more, we have again the aperiodic situation until we fall on a period three zone (for values of $a$ greater than 1.44607), that is, the profit rate has a triennial repetition. In Figure 4, we represent the bifurcation diagram for the profit rate as a function of the parameter $a$.

\section{Kondratiev waves in the profit rate}

If the time unit is long (a decade, a quarter of century, etc.), model (6) exhibits economic cycles that are similar to the Kondratiev waves [1].

Kondratiev waves may be defined as a pattern of regularity characteristic of structural change in the modern world economy in which a cycle is about 50 years long. It consists of an alternation of periods of high growth with others, start-up periods of slower growth. The study of this pattern helps to trace the evolution of the global economy, and aids in politic and economic prediction.

A Kondratiev wave consists of four distinct phases, dramatic mood changes, which one determines the actions of individuals involved in the economy. The awareness of these characteristics allows for the anticipation of the change in the economy and the 


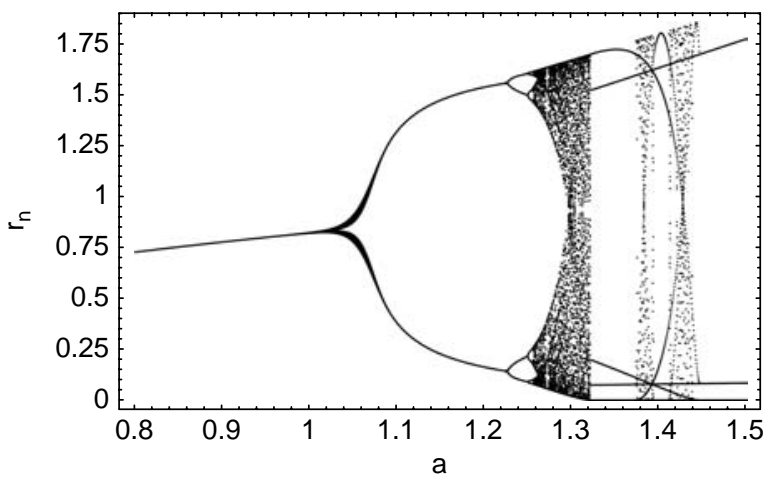

Figure 4. The bifurcation diagram for the profit rate as a function of the parameter $a$.

psychological mood that will prevail. Some analysts compared these four distinct phases in the Kondratiev waves to the seasons: spring (inflationary growth, expansion), summer (stagflation, recession), autumn (deflationary growth, plateau) and winter (depression), that changes each 50 years (Figure 5).

For example, when $a=1.35$ model (6) exhibits the same characteristics as those of the Kondratiev waves (Figure 6). However, the autumn's situation does not permit us to infer any conclusions, because we have first a decline in the profit rate and after that an accelerated growth until the beginning of the winter. This situation is not similar to the

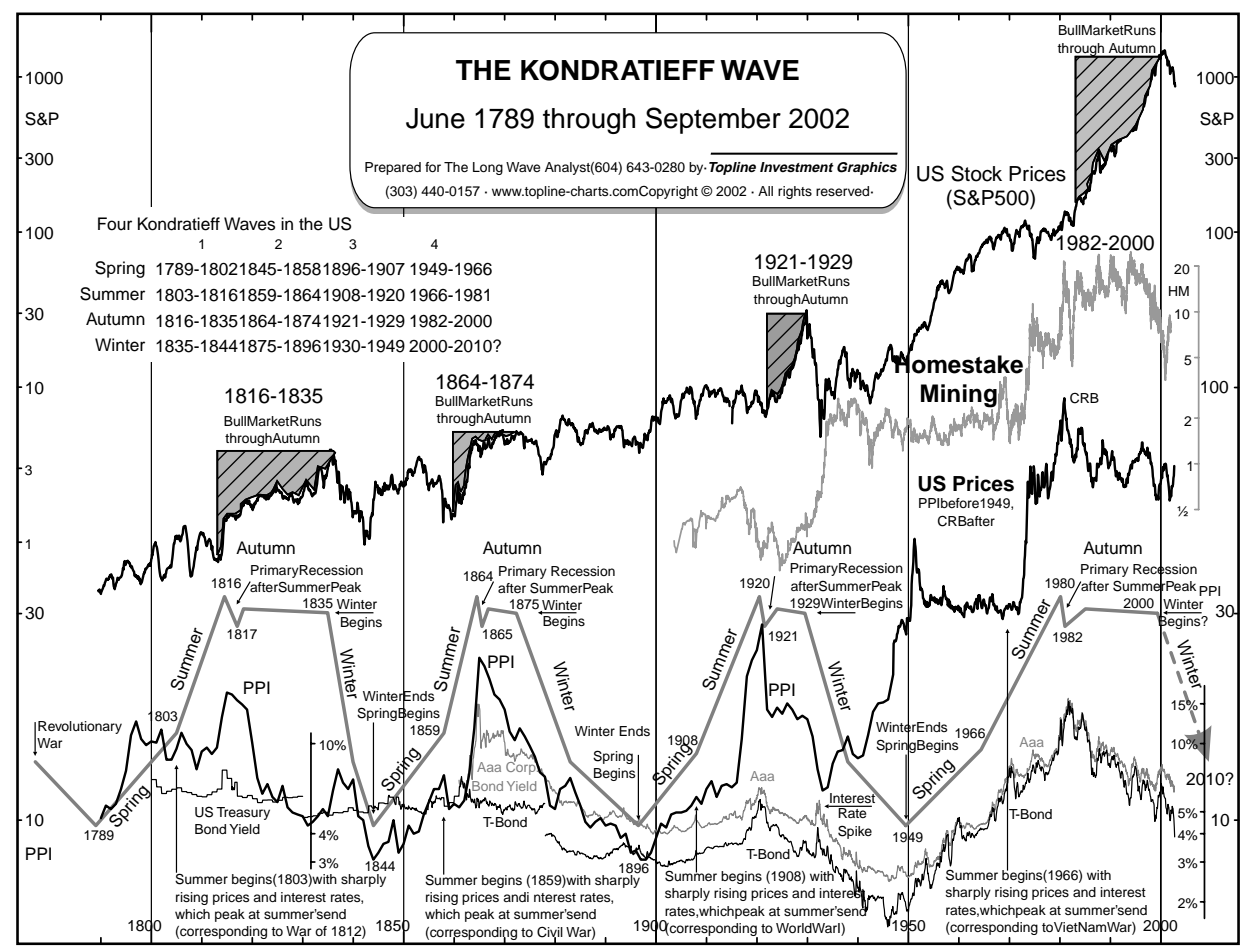

Figure 5. The tendency of global economy according the Kondratiev waves. 


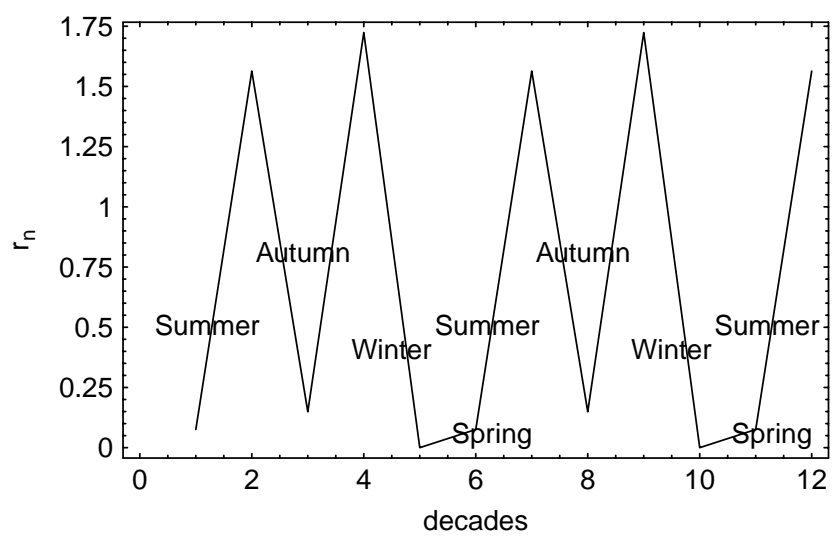

Figure 6. For $a=1.35$ model (6) can be interpreted as the Kondratiev waves. The progression of the profit rate can be viewed in four seasons that repeat each five decades.

Kondratiev waves because there, after the first decline, is a moderate growth rate and then a slow decline until the winter. We believe that this situation in our model is caused by the fact that when the exploitation rate grows, it has a strong influence on the profit rate. That leads to an abrupt variation in the autumn. But this last scenario, in a half of century, represents at least, two or three years. Therefore, the system (6) will adjust itself in the long term.

\section{Conclusion}

In this study, we used the equation that arises from value theory: a purely static equation. That only gives us the fixed relation between the profit rate, the exploitation rate and the organic composition of the capital. That equation is our starting point for several dynamical considerations.

It is obvious that we cannot infer conclusions about the profit rate evolution if we regard this equation as a relation between constants. This is what has been done in the past by economists.

Our goal was to construct an evolutive model in time, that starts in this equation and give it a dynamical interpretation.

Although mathematically possible, that 'law' which arises from Marx's time, that 'the profit rate has a decreasing tendency' has not been proved and it is not a theorem. As always in mathematics, everything depends on the assumptions.

We introduced a unidimensional dynamical system, more realistic than blind previsions based only on immutable laws or in linear approaches.

In this model, we obtain either stability or chaotic behaviour. But whatever the order of the magnitudes of $a$, when we use this last kind of discrete dynamical modulation, the profit rate does not necessarily fall to zero, as predicted by Marx.

Finally, if the scale is a decade, our unimodal model permits to infer long-term cycles in economy.

\section{Note}

1. Email: rafaelluis@netmadeira.com 


\section{References}

[1] N. Kondratieff, The long waves in economic life, Rev. Econom. Statist. XVII(6) (1935), pp. 105-115.

[2] R. Luís, S. Elaydi, and H. Oliveira, An economic model with Allee effect, J. Difference Equ. Appl., 15(8-9) (2009), pp. 877-894.

[3] R. Luís and H. Oliveira, A Dynamical System for a Long Term Economic Model, Proceedings of the Conference ICDEA2007, Lisbon, preprint.

[4] G. Statter, Tendência decrescente da taxa de lucro, Preprint, ISCTE, 2003. 